\title{
The concurrency of several biophysical traits links immunoglobulin light chains with toxicity in AL amyloidosis
}

Luca Oberti $^{1}$, Martina Maritan ${ }^{1}$, Paola Rognoni ${ }^{2}$, Alberto Barbiroli ${ }^{3}$, Francesca Lavatelli ${ }^{2}$, Rosaria Russo $^{4}$, Giovanni Palladini ${ }^{2}$, Martino Bolognesi ${ }^{1}$, Giampaolo Merlini ${ }^{2}$ and Stefano Ricagno ${ }^{1}$

${ }^{1}$ Department of Bioscience, University of Milan, Italy,

${ }^{2}$ Amyloidosis Research and Treatment Center, Fondazione IRCCS Policlinico San Matteo, and Department of Molecular Medicine, University of Pavia, 27100, Pavia, Italy,

${ }^{3}$ DeFENS - Dep. of Food, Environmental and Nutritional Sciences, University of Milan, Italy,

${ }^{4}$ Department of Physiopathology and Transplantation, University of Milan, Italy

Address for correspondence: Stefano Ricagno, Department of Bioscience, University of Milan, Via Giovanni Celoria 26, 20133, Milan, Italy. E-mail: stefano.ricagno@unimi.it

Background. Light chain amyloidosis (LC-AL) is the most common systemic amyloidosis. It is caused by the overproduction and the aggregation of toxic and monoclonal immunoglobulin light chains (LC) in target organs. Among all the organs injured by the pathology, the heart is the most affected one. In particular, the ventricular compliance is reduced, resulting in a symptomatic congestive heart failure [1]. It is extremely relevant that due to the genetic rearrangement and somatic hypermutation, a high variability among LCs' sequences is generated. This means, virtually, that each AL patients present different amyloidogenic LCs [2], stressing in the necessity to investigate a large set of cases. To date, it is particularly interesting the observation described in Milani et al., 2018 [1], where it was observed that the severity of heart symptoms is linked with the blood concentration of full-length LCs, which are the major circulating species. Specifically, these facts highlight a crucial role for full-length LC in the pathology beyond the amyloid deposits. However, up to now, despite the several works conducted, the molecular bases of proteotoxicity and the aggregation mechanism(s) are still unclear.

Material and methods. Our approach consisted in a biophysical and structural characterization. LCs were purified from patients' urine, or by the recombinant expression in E.coli [3]. Protein stability was evaluated by circular dichroism (CD) and by anilinonaphthalene-1-sulfonic acid (ANS) fluorescence, while flexibility and dynamics was studied by limited proteolysis [3]. The Xray diffraction experiments, on LCs crystals, were carried out at the European Synchrotron Radiation Facility (ESRF) in Grenoble, France.

Results. In order to achieve as generalizable as possible data, our study is based on a large pool of thirteen $\lambda$ full-length LCs divided in two groups. In particular, eight LCs are amyloidogenic and responsible of severe cardiac symptoms in AL patients (H LCs); five are non-amyloidogenic LCs and selected from patients affected by multiple myeloma, and used as control (M LCs). In order to highlight potential determinants of LCs proteotoxicity and aggregation, all proteins were extensively structurally and biophysically characterized. Firstly, X-ray diffraction of seven LCs crystals (five H LCs and two M LCs) reveals that H and M LCs structures match very closely. From the biophysical point of view, the melting temperature measurements assessed by three independent spectroscopic techniques demonstrate that H LCs tend to be less stable than M LCs (fig. 1A). Moreover, limited proteolysis by trypsin and protease $\mathrm{K}$ strongly indicate that a more pronounced flexibility for H LCs compared to M LCs (fig. 1B). These findings suggest thermodynamic properties and protein dynamics to have a role in the molecular proteotoxicity mechanism(s). In particular, since the crystal structures are conserved, it is very likely that dynamics plays a critical role in defining the propensity of a LC to be toxic or not. 
Recently, Diomede et al., 2017 [4] described H LCs toxicity in vivo to be increased by the presence of copper ions. Along this line, we are investigating potential interactions between LCs and bivalent cationic metal ions, including copper. In particular, we observe a direct and specific binding between $\mathrm{H} \mathrm{LCs}$ and $\mathrm{Cu}^{2+}$. Our findings show that the LC-copper interaction destabilizes the proteins and it seems to turn H LCs in a more flexible state, underling the importance of dynamics in determining LC toxicity.
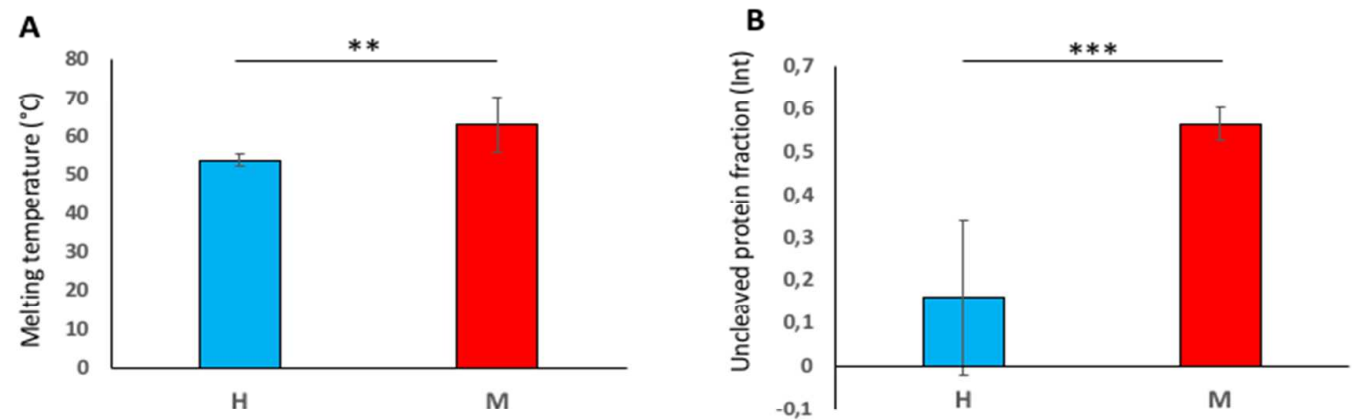

C
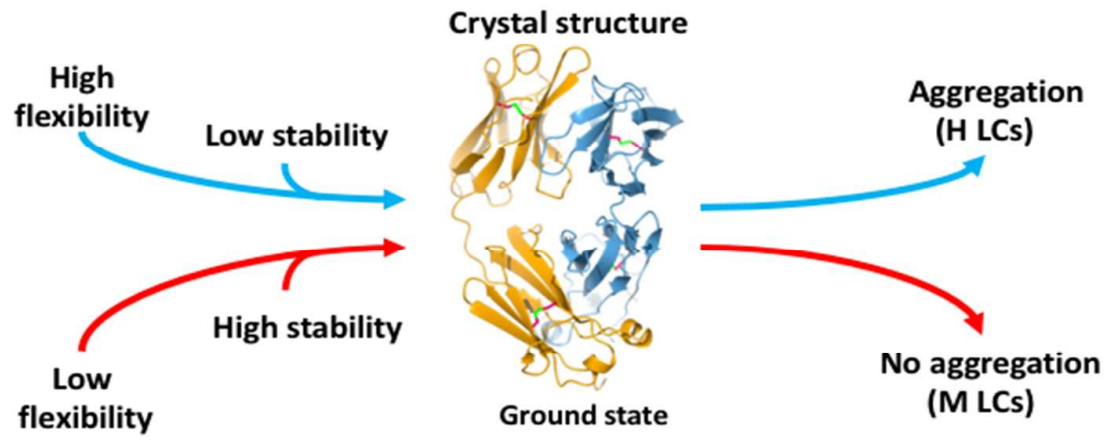

Figure 1. A. Average of H LC and M LC melting temperatures. B. Average of the $\mathrm{H}$ and M uncleaved protein fraction at $180 \mathrm{~min}$ after limited proteolysis; *** $p$-value $<0.0005$; ** $p$-value $<0.005$. C. Graphical representation of the biophysical properties contributing in the amyloidogenic properties.

Discussion and conclusions. The new contribute of this work highlights the concurrency of different biophysical traits to be linked with LCs amyloid propensity. Our data suggest that thermal stability and flexibility/dynamics correlate with the proteotoxicity LCs tendency, whereas the overall structural determinants are conserved between H and M LCs (fig. 1C).

Declaration of interest. The authors report no conflict of interest.

\section{References}

1. Milani, P. et al. Light Chain Amyloidosis. Mediterranean Journal of Hematology and Infectious Diseases. 2018;10:e2018022.

2. Merlini G, Palladini G. Light chain amyloidosis: the heart of the problem. Haematologica. 2013; 98:1492-5.

3. Oberti, L. et al. Concurrent structural and biophysical traits link with immunoglobulin light chains amyloid propensity. Scientific reports. 2017; 7.1.

4. Diomede, L. et al. Cardiac light chain amyloidosis: the role of metal ions in oxidative stress and mitochondrial damage. Antioxidants \& redox signalling. 2017; 27.9: 567-582. 